Ann. Biol. anim. Bioch. Biophys., I970, 10 (4), 581-587.

\title{
INFLUENCE DU LACTATE ET DU NAD SUR LE TRANSPORT DU GLUCOSE PAR L'INTESTIN RETOURNÉ DE RAT
}

\author{
F. FAUDEMAX, R. ROZEN et Cl. SAUTIER \\ avec la collaboration technique de $\mathrm{R}$. Chabbert \\ Laboratoire de Nutrition Humaine, INSERM (UI) \\ Professeur J. Trémolières, Hôpital Bichat, Paris $18 \mathrm{e}$
}

RÉSUMÉ

Nous avons étudié le transport intestinal de glucose sur des segments de jéjunum de rat, et le rôle du lactate de sodium et du NAD sur ce transport. La méthode utilisée est celle de CRANE et WiLson d'anses retournées. Le milieu physiologique d'incubation est le liquide de Robinson. Les variations de pH, de sodium, de potassium, et de glucose sont mesurées, dans les milieux extérieur et intérieur à l'anse.

Par comparaison avec les anses témoins, l'addition simultanée de lactate de sodium et de NAD au liquide en contact avec la muqueuse augmente significativement le transport du glucose, tandis que les concentrations de sodium ne varient guère. L'influence du pH du milieu d'incubation est discutée.

L'interprétation de nos résultats conclut au rôle des systèmes d'oxydo-réduction cellulaires sur le transport du glucose.

\section{INTRODUCTION}

Nous avons précédemment montré (FAUDEMAY et al., I968; ROZEN, I968) un accroissement du transport du glucose en présence d'acide lactique, sous oxygène par l'anse intestinale retournée de rat.

D'autre part, nous avons mis en évidence (GRIFFATON et al., I969) une augmentation $d u$ transport $d u$ glucose par l'anse intestinale retournée du rat ayant reçu une injection intra-péritonéale, de trois fois $2 \mathrm{~g} / \mathrm{kg}$, d'éthanol, ce transport ne s'accroissant plus si on ajoute au milieu de l'acide lactique.

L'éthanol provoquant une augmentation du rapport NADH/NAD dans le foie de rat (FORSANDER et al., I958 ; BARON et al., I968), nous avons pensé que le transport actif du glucose pourrait être en relation avec les variations d'équilibre des systèmes d'oxydo-réduction. 
Pour vérifier cette hypothèse, il nous a paru logique de faire intervenir le NAD directement au contact de la muqueuse et d'observer son effet sur le transport du glucose.

\section{MATÉRIEI, ET MÉTHODES}

La technique utilisée est celle décrite précédemment (Griffaton et al., I969). On prélève quatre segments égaux d'intestin grêle retrourné de $7 \mathrm{~cm}$ de long que l'on fixe sur l'appareil de CRANE et Wilson (1958). On les remplit de I,5 ml de liquide de Robinson préalablement oxygéné pendant $\mathrm{I}_{5}$ minutes $\left(\mathrm{O}_{2}\right.$ I $00 \mathrm{p}$. Ioo). Chaque anse est incubée dans ${ }^{7} 7 \mathrm{ml}$ de liquide de Robinson additionné respectivement des substrats suivants :

- glucose (milieu " $\mathrm{RG}$ ")

- glucose + lactate de sodium (milieu " RGL ")

- glucose + NAD + nicotinamide (milieu " RG-NAD »), la nicotinamide servait de stabilisateur du NAD

- glucose + lactate de sodium + NAD + nicotinamide (milieu " RGL-NAD»).

Les concentrations des substrats sont :

- glucose : Io $\mathrm{mM}$

- lactate de sodium : $20 \mathrm{mM}$

- NAD : I $\mathrm{mM}$

- nicotinamide : $40 \mathrm{mM}$ nutes.

Les milieux d'incubation sont oxygénés $\left(\mathrm{O}_{2}\right.$ Ioo p. Ioo) et maintenus à $37^{\circ}$ pendant 60 mi-

A la fin de l'expérience, on recueille le liquide à l'intérieur de l'anse (liquide "séreux") et à l'extérieur (liquide "muqueux").

Dans ces deux milieux, on dose alors :

- le glucose par la méthode enzymatique de HugGetT et Nixon (1957).

- Le sodium et le potassium au photomètre de flamme (Électro-synthèse).

On détermine le $\mathrm{pH}$ (pHmètre E. I. L.).

\section{RÉSULTATS}

\section{A. - Transport du glucose}

Les résultats sont présentés dans le tableau I exprimant les concentrations de glucose dans les milieux muqueux et séreux avant et après incubation. Les comparaisons les plus intéressantes portent sur les concentrations de glucose apparu du côté séreux c'est-à-dire sur le glucose transporté :

Lorsqu'on ajoute du lactate de sodium et du NAD au liquide muqueux le transport du glucose est augmenté significativement par rapport aux milieux qui contiennent du glucose seul (RG) ou du glucose et du lactate de sodium (RGL) ou encore du glucose et du NAD (RG-NAD).

\section{B. - Le sodium et le potassium}

La concentration de sodium ne varie pas significativement dans les liquides séreux et muqueux après 60 minutes (I50 méq $\pm+I, 30$ ). Par contre, celle du potassium 


\section{TABLEAU I}

Effets du lactate de sodium et du NAD (土 nicotinamide) ajoutés au milieu d'incubation, sur l'absorption du glucose par une anse intestinale retournée de rat

\begin{tabular}{|c|c|c|c|c|}
\hline \multirow{2}{*}{ Milieu d'incubation } & \multicolumn{2}{|c|}{ Côté muqueux (M) } & \multirow{2}{*}{$\frac{\text { Côté séreux (S) }}{\text { Glucose }}$} & \multirow{2}{*}{$\frac{\mathrm{S}}{\mathrm{MI}}\left(\begin{array}{ll}60 \mathrm{mn}) \\
(60 \mathrm{mn})\end{array}\right.$} \\
\hline & $\begin{array}{l}\text { Glucose } \\
\text { au temps } 0\end{array}$ & $\begin{array}{c}\text { Glucose } \\
\text { au temps } 60 \mathrm{mn}\end{array}$ & & \\
\hline $\mathrm{RG}$ & $10,86 \pm 0,150$ & $8,40 \pm 0,150$ & $9,16 \pm 0,9^{\prime} 0$ & 1,09 \\
\hline RGL & $10,70 \pm 0,150$ & $8,39 \pm 0,150$ & $9,90 \pm 0,940$ & 1,18 \\
\hline RG-NAD & $10,81 \pm 0,150$ & $8,59 \pm 0,150$ & $9,41 \pm 0,910$ & 1,10 \\
\hline RGL-NAD & $10.53 \pm 0.150$ & $8,24 \pm 0,150$ & $12,52 \pm 0,940$ & 1,52 \\
\hline
\end{tabular}

Résultats exprimés en concentration de glucose ( $\mu \mathrm{mol} / \mathrm{ml}), \mathbf{M} \pm s_{\mathbf{M}}$ (résultats de 9 exp.).

TABLEAU 2

Concentration du potassium au début et à la fin de l'expérience dans les liquides muqueux (M) et séreux (S)

p. Ioo de variations $\left(\frac{\left[\mathrm{C}_{0}\right]-\left[\mathrm{C}_{60}\right]}{\left[\mathrm{C}_{60}\right]}\right) \times$ Ioo

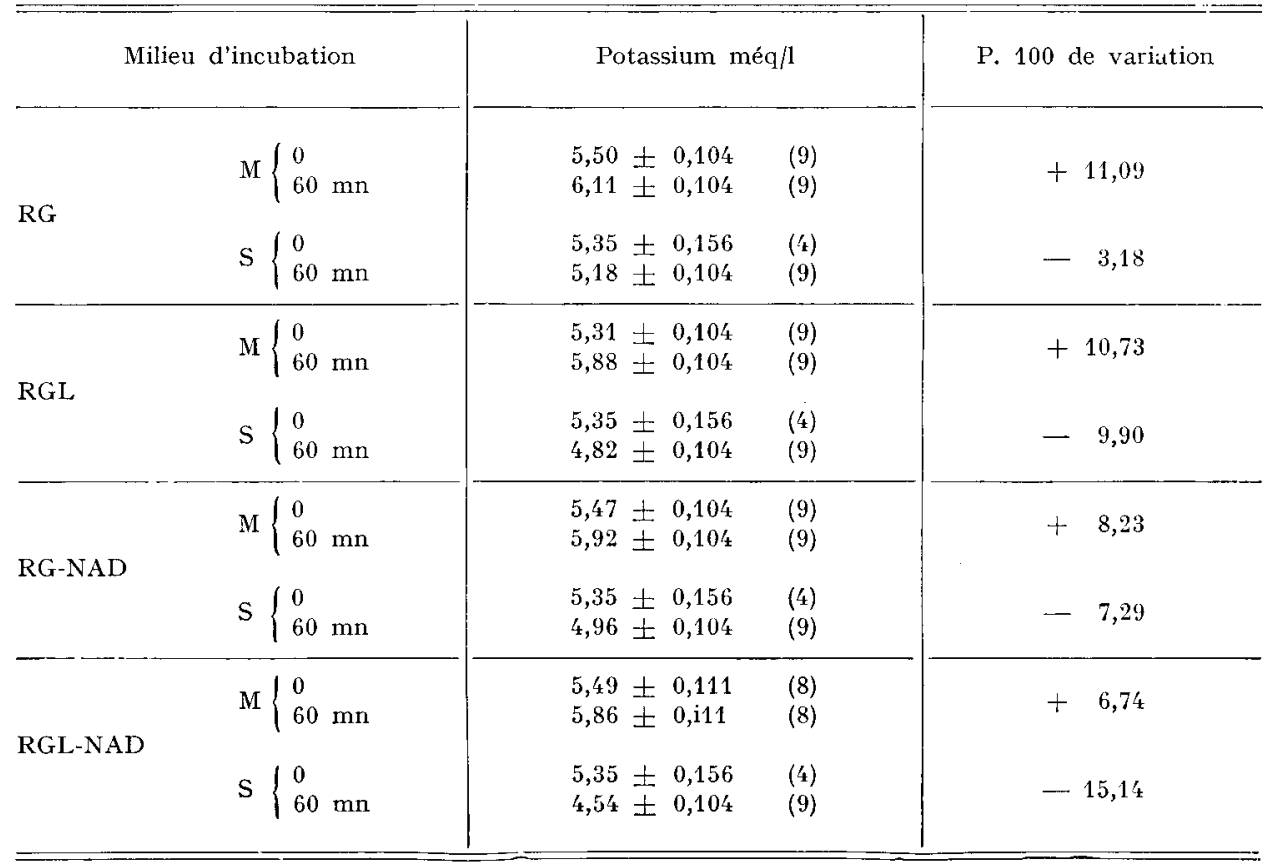


(tabl. 2) s'élève du côté muqueux et diminue du côté séreux. Le pourcentage de variation calculé selon la formule suivante :

$$
\begin{gathered}
\frac{\left[\mathrm{C}_{0}\right]-\left[\mathrm{C}_{60}\right]}{\left[\mathrm{C}_{60}\right]} \times \text { I00 } \\
\mathrm{C}_{0}=\text { concentration du potassium au temps o } \\
\mathrm{C}_{60}=\text { concentration du potassium au temps } 60
\end{gathered}
$$

nous montre une augmentation d'environ ro $\mathrm{p}$. Ioo du côté muqueux et une diminution d'environ ro p. Ioo du côté séreux.

$$
\text { C. - Le } p H
$$

Nous avons étudié le $\mathrm{pH}$ du côté muqueux avant et après incubation et les résultats sont exprimés dans le tableau 3.

Nous n'avons pu faire de mesures dans le liquide séreux, son volume étant insuffisant.

\begin{tabular}{|c|c|c|c|c|}
\hline \multirow{2}{*}{ Temps } & \multicolumn{4}{|c|}{ Milieux d'incubation } \\
\hline & $R G$ & $\mathrm{RGL}$ & $\mathrm{RG}-\mathrm{N} A \mathrm{D}$ & RGL-NAD \\
\hline $\mathbf{0}$ & 7,20 & 7,16 & 6,72 & 6,66 \\
\hline $60 \mathrm{mn}$ & 6,65 & 6,65 & 6,43 & 6,19 \\
\hline
\end{tabular}

TABLEAU 3

$$
p H \text { du milieu muqueux avant et après incubation de } 60 \text { minutes }
$$
d'une anse intestinale retournée de rat

\section{DISCUSSION}

\section{A. - Caractéristiques du milieu d'incubation}

a) Le $p H$.

Le pH de la solution de Robinson glucosée est au temps o de 7,2. L'addition de lactate de sodium ne fait pas varier le $\mathrm{pH}$ tandis, que l'addition de NAD 1'abaisse légèrement.

Le rôle du $\mathrm{pH}$ de la solution d'incubation sur le transport du glucose a été étudié par de nombreux auteurs. PONZ et LARRALDE (I95I) trouvent un transport maximum pour un $\mathrm{pH}$ compris entre 6,5 et 7. Cependant IIDA et coll. (Ig68) sur des sacs retournés d'intestin grêle de hamster observent un transport maximum à $\mathrm{pH} 5,9$.

Dans les deux milieux où nous avons ajouté du NAD, le pH est sensiblement identique alors que le transport est plus grand dans le milieu RGL-NAD que dans le milieu RG-NAD. De plus, dans les deux milieux où le $\mathrm{pH}$ est légèrement supérieur à 7 (RG et RGL) le transport du glucose est semblable à celui où le pH est de 6,66 (milieu RG-NAD). 
Ce n'est donc pas le $\mathrm{pH}$ initial du milieu d'incubation qui peut rendre compte des variations de transport que nous avons observées.

b) Le sodium et le potassium.

L'absence de variation de la concentration du sodium peut s'expliquer par le double flux du sodium. Pour voir une variation dans le flux net, il faudrait utiliser du sodium marqué.

La variation de concentration du potassium (tabl. 2) dans les deux compartiments muqueux et séreux peut s'expliquer de plusieurs façons :

Du côté muqueux, la desquamation des cellules épithéliales de la muqueuse, riches en potassium, produit en partie l'augmentation du potassium. D'autre part, cette augmentation peut être la conséquence d'un mouvement d'eau du côté muqueux vers le côté séreux pendant le transport du glucose.

Du côté séreux, la dilution consécutive à ce mouvement d'eau diminue la concentration du potassium. Les variations de concentration du potassium seraient dues à des mouvements d'eau et non à des transferts de potassium.

Nous n'avons pas mesuré dans ces expériences le volume de liquide retrouvé du côté séreux après les 60 minutes d'incubation à cause de l'importance de l'erreur dans cette détermination. En effet, la quantité de liquide séreux recueillie est très imprécise : la variation du volume d'eau est très faible par rapport aux causes d'erreur (difficulté de recueil, imbibition du tissu...). RozEN (communication personnelle) utilisant une technique analogue mais des anses plus longues, mesure la variation de l'eau à l'intérieur de l'anse et trouve une augmentation de 15 p. Ioo ce qui corrobore l'hypothèse d'une dilution du potassium dans le milieu séreux.

En admettant cette hypothèse, nous avons calculé le glucose transporté au bout de 60 minutes (tab1. 4) en tenant compte du facteur de dilution calculé à partir du potassium (tabl. 2 ; colonne p. Ioo de variation). Nous utiliserons lors de la discussion sur le transport du glucose, ces valeurs corrigées.

TABLEAU 4

Concentration du glucose en umol/ml (Séreux $60 \mathrm{mn}$ ) et rapport $S / M$ calculés après correction par le facteur de dilution du polassium

\begin{tabular}{|c|c|c|}
\hline Milieu d'incubation & Séreux $60 \mathrm{mn}$ & Séreux/muqueux $(\mathrm{S} / \mathrm{M})$ \\
\hline$R G \ldots$ & 9,45 & 1,12 \\
\hline RGL $\ldots \ldots \ldots \ldots$ & 10,88 & 1,30 \\
\hline RG-NAD $\ldots$. & 10,10 & 1,17 \\
\hline RGL-NAD $\ldots \ldots \ldots$ & 14,42 & 1,75 \\
\hline
\end{tabular}

c) Nicotinamide.

Nous nous sommes placés dans des conditions de grand excès de nicotinamide afin d'empêcher la destruction du NAD, comme l'ont proposé MANN et QUASTEI (I94I). 
Nous avons fait des expériences en ajoutant du NAD sans nicotinamide aux milieux RG et RGL : les concentrations de glucose transporté sont respectivement de 9,79 et 8,3I, non différentes significativement des résultats trouvés dans les milieux RG et RGI. I a nicotinamide est donc bien indispensable pour préserver la molécule de NAD.

L'hyperosmolarité du côté muqueux apportée par la nicotinamide pourrait provoquer un appel d'eau de ce côté. Mais comme nous l'avons vu, dans tous les milieux y compris ceux contenant de la nicotinamide, l'augmentation de concentration du potassium du côté muqueux s'explique par un mouvement d'eau en sens inverse, du compartiment muqueux vers le compartiment séreux. D'autre part, l'examen du tableau I ne conduit pas à donner un rôle important à l'hyperosmolarité provoquée par la nicotinamide : en effet, les rapports séreux/muqueux finaux en milieu RG et en milieu RG-NAD sont identiques alors que ce rapport est nettement augmenté dans le milieu RGI-NAD. La présence de nicotinamide n'intervient donc pas dans le transport du glucose.

\section{B. - Transport du glucose}

Nous avions émis l'hypothèse que le lactate de sodium agissait sur le transport du glucose en augmentant le rapport NADH/NAD.

D'après nos résultats, seul le lactate additionné de NAD provoque une augmentation significative du transport du glucose (tabl. I). Les résultats sont encore plus nets si l'on tient compte de la dilution dans le milieu séreux, calculés à partir des variations de concentration du potassium (tabl. 4) : le rapport S/M qui exprime le pouvoir de concentration de la muqueuse, très nettement supérieur à $I(I, 30)$ en présence de lactate, augmente encore quand le lactate est additionné de $\operatorname{NAD}(I, 75)$.

Ie NAD seul (RG-NAD) n'a pas d'action; on peut donc penser que le lactate apporte une grande quantité d'ions $\mathrm{H}$ qui provoque la formation d'un excès de $\mathrm{NADH}$, d'où une augmentation très importante du rapport $\mathrm{NADH} / \mathrm{NAD}$. Cette action $\mathrm{du}$ rapport $\mathrm{NADH} / \mathrm{NAD}$ sur le transport s'expliquerait par une activation de la chaîne des transporteurs d'H, celle-ci augmentant la formation d'ATP.

Mais, on peut concevoir que le lactate additionné de NAD agit comme fournisseur de pyruvate qui entre directement dans le cycle de Krebs. Or, WORBE et DuGarDIN (I967) étudiant l'effet de l'acide pyruvique sur la glycolyse dans la muqueuse intestinale de rat montrent que le pyruvate n'augmente pas le métabolisme oxydatif.

Nous pensons que l'augmentation du transport du glucose s'effectue par l'interdiaire du NADH.

Ceci est à rapprocher des travaux de Conway (I963) qui lie le transport du sodium aux modifications de la chaîne des transporteurs d'électrons et pour qui l'énergie peut dériver indirectement de la fermentation de l'acide lactique.

En conclusion : 'une augmentation significative du transport du glucose est observée en présence de lactate et de NAD. Parmi les hypothèses envisagées pour expliquer cette action, le rôle de $\mathrm{NADH}$ formé ainsi, semble la plus probable. 


\section{REMERCIEMENTS}

Travail subventionné en partie par la Fédération Internationale Laitière.

\section{SUMMARY}

\section{EFFECT OF IACTATE AND NAD ON GLUCOSE TRANSPORT BY THE EVERTED RAT INTESTINE}

The transport of glucose by the jejunal loop of rat intestine and the part played in the transport by sodium lactate and NAD were studied using Crane and WILson's method of everted loop and Robinson's liquid as physiological medium. Variations in pIf, sodium, potassium and glucose concentrations were measured both in the internal and external media.

Compared to a control loop, simultaneously adding sodium lactate and NAD to the medium in contact with the muccus membrane significantly increased glucose transport, whereas sodium concentrations scarcely vary. Variations in potassium concentrations are interpreted in terms of fluide movements and compared with glucose transport under the various experimental conditions. The effect of $\mathrm{pH}$ of the incubation medium is discussed.

Our findings lead us to the view that cell oxydo-reduction mechanisms can effect glucose transport.

\section{RÉFÉRENCES BIBLIOGRAPHIQUES}

Baron P., Griffaton G., Lowy R., ig68. itude comparée des perturbations métaboliques provoquées dans le foie du rat par administration d'éthanol ou d'autres composés. I. Cas d'une injection intrapéritonéale d'éthanol. Fd. Cosmet. Toxicol., 6, 729-736.

Conway li. J., I963. Significance various factors including lactic-dehydrogenase on the active transport of sodium ions in skeletal muscle. Nature, $98,760-763$.

CRANE R. K., Wilson T. H., I958. In vitro method for the study of the rate of intestinal absorption of sugars. J. Appl. Physiol., 12, I $45-\mathrm{r}_{4} 6$.

Faudemay F., Griffaton G., Sautier Cl., Trémolikes J., rg68. Acide lactique et absorption intestinale du glucose. Étude in vitro sur l'intestin retourné de rat. Ext. Écon. Méd. anim., 6, 379-384.

Forsander O. A., Raiha N. C. R., Suomalainen H., i958. Alkoloxydation und Bildung von acetoacetat in normaler und glycogen armer intakter Rattenleber. Hope-Seyler's $Z$. Physiol. Chem., 312, 243 .

Griffaton G., Faudemay F., Sautier Cl, I_owy R., I969. Absorption du glucose et de l'acide lactique par l'anse retournée de rat à la suite d'injections intrapéritonéales d'éthanol. Nutr. Dieta, 11, 259-269.

Huggett A. St G., Nixon D. A., 1957. Use of glucose-oxydase, peroxydase and o-dianisidine in determination of blood and urinary glucose. Lanc, ii, 368 .

Iida H., Moore E. W., Brortman S. A., Zamcheck N., rg68. Effect of $\mathrm{pH}$ on active transport of $d$-glucose in the small intestine of hamsters. Proc. Soc. Exp. Biol. and Med., 127, 730-732.

Mann P. J. G., Quastel. J. H., I94I. Nicotinamide, cozymase and tissue metabolism. Biol. J., 35, 502 .

Ponz F., L.ARralde J, I95I. Effect of phosphates and the pH on sugar absorption. Nature, 168, 912.

Rozen R., I968. Influence de l'acide lactique sur l'activité métabolique de l'intestin grêle de rat. Ann. Nutr. Alim., 22, I55-I68.

Worbe J. F., Dugardin M. C., I967. Activité glycolytique de la muqueuse intestinale du rat. Action de l'acide pyruvique. J. Physiol., 59, 532. 